\title{
Radiation Pattern Reconfigurable Antenna for IoT Devices
}

\author{
Zakaria Mahlaoui $\mathbb{D}^{1,2}{ }^{1,2}$ Eva Antonino-Daviu $\mathbb{D}^{1}{ }^{1}$ and Miguel Ferrando-Bataller $\mathbb{D}^{1}$ \\ ${ }^{1}$ Instituto de Telecomunicaciones y Aplicaciones Multimedia, Universitat Politècnica de València, Valencia, Spain \\ ${ }^{2}$ National School of Applied Sciences, Cadi Ayyad University, Marrakesh, Morocco
}

Correspondence should be addressed to Eva Antonino-Daviu; evanda@upvnet.upv.es

Received 11 February 2021; Revised 1 July 2021; Accepted 7 August 2021; Published 18 August 2021

Academic Editor: Giorgio Montisci

Copyright (c) 2021 Zakaria Mahlaoui et al. This is an open access article distributed under the Creative Commons Attribution License, which permits unrestricted use, distribution, and reproduction in any medium, provided the original work is properly cited.

\begin{abstract}
Based on the characteristic mode theory, a versatile radiation pattern reconfigurable antenna is proposed. The analysis starts from two parallel metallic plates with the same and different dimensions. By means of two PIN diodes, the size of one of the parallel metallic plates can be modified and consequently the behavior of the radiation pattern can be switched between bidirectional and unidirectional radiation patterns. Moreover, a SPDT switch is used to adjust the frequency and match the input impedance. The reconfigurable antenna prototype has been assembled and tested, and a good agreement between simulated and measured results is obtained at $2.5 \mathrm{GHz}$ band which fits the IoT applications.
\end{abstract}

\section{Introduction}

As a fundamental part of a wireless system and due to the strong demand of the market for diverse applications, antennas are sometimes required to change their basic characteristics with respect to the application needs. To fulfil this expectation, CM (characteristic mode) theory [1-3], which is a relatively new approach in the antenna field, presents an easy way to understand the physics behind several antenna features, such as the radiation pattern shape, beamwidth and direction, polarization, and bandwidth and operating frequency. This physical understanding provides information about how modes operate and helps in the practical antenna design process, making it easier $[4,5]$.

The huge growth of Internet of Things (IoT) and smart industrial applications builds up many engineering and scientific challenges that involve ingenious research efforts from both academic and industrial society for the development of cost-effective, scalable, efficient, and reliable antenna systems for IoT. This is why reconfigurable antennas (RAs) are conceived as a suitable system that can change its basic characteristics [6, 7] such as the operating frequency $[8,9]$, the radiation pattern $[10,11]$, or even the polarization $[12,13]$ to cope with the demand of several communication systems such as IoT sensors and mobile stations [14]. Reconfigurable antennas are classified into several types, depending on the use of semiconductors such as PIN diodes [15] and varactor diodes [11, 16], RF MEMS [17], photoconductive components [18], and reconfiguration by changing material properties [19] or by mechanical handling [20].

This paper describes a radiation pattern reconfigurable antenna based on the use of two rectangular parallel plates. The design is based on that presented in [21], but a significant improvement has been provided with the current design. Firstly, the geometry is proposed and a CM analysis (CMA) is performed in order to explain the behavior of the antenna from a modal perspective. Then, a reconfigurable radiating structure using PIN diodes and a SPDT switch [22] is proposed.

The goal of the proposed design is to switch between a bidirectional and unidirectional radiation pattern, depending on the state of the PIN diodes. The aim of using a SPDT switch is to reach a common operating frequency $(2.5 \mathrm{GHz})$ at both states, avoiding the modification of the basic geometry. Hence, the proposed antenna proposes a convenient solution for several IoT applications, as it can produce different beam conditions in a sensor without changing physically the antenna structure. 
CMA, antenna simulation, and design of the structure have been performed by means of FEKO [23] and CST Microwave Studio simulation software applications [24].

The paper starts with a brief overview of the CM theory and the physical interpretation of the modal parameters. Then, the general procedure for designing reconfigurable antennas using CMA is discussed, following the CMA of two rectangular parallel plates. In Section 5, a radiation pattern reconfigurable antenna is proposed, and a prototype and measurements are presented in the next section. Finally, conclusions are discussed.

\section{Characteristic Mode Overview}

The CM theory was initially developed by Garbacz in 1968 [1] and later refined by Harrington and Mautz in 1971 [3]. This theory has recently become a versatile modal analysis tool for antennas with arbitrary shapes and materials. The CMs together with their metric parameters provide the following effective information for antenna analysis and design:

(i) Resonant frequencies of the dominant mode and higher-order modes.

(ii) Modal radiation fields in the far-field range.

(iii) Modal currents on the surface of the analyzed structure.

(iv) Significance of the modes at a given frequency.

CMs are defined as a set of orthogonal radiating current modes that are supported by a conducting or dielectric surface. A weighted eigenvalue equation is derived from the method of moments (MoM) impedance matrix $[3,5,25]$, and a set of orthogonal eigencurrents (i.e., CMs), together with their associated eigenvalues, is obtained. Due to the orthogonality of the eigencurrents, the total current on the surface of the conductor can be expanded into a set of modes. Eigenvalues provide information about the radiating behavior of the associated mode. Moreover, attributes such as the characteristic angle or modal significance can be calculated, which are associated to the eigenvalue.

In the following, a brief review of the parameters associated to the CMA will be provided, in order to obtain physical insight into the radiating behavior of an antenna with arbitrary shape.

2.1. Physical Interpretation of the Eigenvalue. Based on the approach developed in $[3,5]$, CMs can be obtained as the eigenfunctions of the following particular weighted eigenvalue equation:

$$
X J_{n}=\lambda_{n} R J_{n}
$$

where $J_{n}$ and $\lambda_{n}$ are the real eigenvectors and eigenvalues, respectively, $n$ is the index of the order of each mode, and $R$ and $X$ are the real and imaginary parts of the impedance matrix of the MoM. The electric fields $E_{n}$ and the magnetic fields $H_{n}$ produced by characteristic currents $J_{n}$ on the surface of a conducting body $S$ are called characteristic fields or eigenfields associated to $J_{n}[3]$.

As presented in [3], the selection of $R$ as a weight operator in equation (1) is responsible for the orthogonality properties of characteristic modes, expressed by (2) and (3):

$$
\begin{aligned}
& \left\langle\overrightarrow{J_{m}}, R \cdot \overrightarrow{J_{n}}\right\rangle=\left\langle\overrightarrow{J_{m}^{*}}, R \cdot \overrightarrow{J_{n}}\right\rangle=\delta_{m n}, \\
& \left\langle\overrightarrow{J_{m}}, X \cdot \overrightarrow{J_{n}}\right\rangle=\left\langle\overrightarrow{J_{m}^{*}}, X \cdot \overrightarrow{J_{n}}\right\rangle=\lambda_{n} \delta_{m n},
\end{aligned}
$$

where

$$
\delta_{m n}= \begin{cases}1, & \text { if } m=n, \\ 0, & \text { if } m \neq n .\end{cases}
$$

Choosing $m=n$ gives the following physical interpretations of the eigenvalues [5]:

(i) The total stored field energy within a radiation or scattering problem is proportional to the magnitude of the eigenvalues.

(ii) The case of $\lambda_{n}=0$ corresponds to the case of resonance, and the associated modes are known as the resonant modes.

(iii) In the case of $\lambda_{n}>0$, the stored magnetic field energy dominates over the stored electric field energy, so the associated modes are known as the inductive modes.

(iv) In the case of $\lambda_{n}<0$, the stored electric field energy dominates over the stored magnetic field energy, so the associated modes are known as the capacitive modes.

2.2. Physical Interpretation of the Modal Significance. The modal significance $\left(\mathrm{MS}_{n}\right)$ is a function of the operating frequency and the dimensions of the conducting object [26]. It measures the contribution of each mode to the total electromagnetic response to a given source. As can be observed in equation (5), $\mathrm{MS}_{n}$ transforms the $[-\infty,+\infty]$ value range of eigenvalues $\lambda_{n}$ into a much smaller range of $[0,1]$. In many cases, it is more convenient to use the $\mathrm{MS}_{n}$ other than $\lambda_{n}$ to investigate the resonant behavior across a wide frequency band.

$$
\mathrm{MS}_{n}=\left|\frac{1}{1+j \lambda_{n}}\right| .
$$

In addition, $\mathrm{MS}_{n}$ also provides a convenient way to measure the bandwidth $\left(\mathrm{BW}_{n}\right)$ of each $\mathrm{CM}$. This bandwidth can be defined as the frequency range where the power radiated by the mode is more than one-half of the power radiated by the same mode at resonance and can be expressed as shown in the following equation:

$$
\mathrm{MS}_{\mathrm{HP}_{n}}=\left|\frac{1}{1+j \lambda_{n}}\right|=\frac{1}{\sqrt{2}}=0.707 \text {. }
$$

Half power (HP) at resonance corresponds to a cutback of the normalized current by a factor $\sqrt{2}$ [5]. Moreover, $\mathrm{MS}_{n}$ 
is also used to identify the significant modes and nonsignificant modes. CMs with $\mathrm{MS}_{n} \geq 0.707$ are referred to as significant modes, whereas CMs with $\mathrm{MS}_{n}<0.707$ are referred to as nonsignificant modes.

\subsection{Physical Interpretation of the Characteristic Angle.} The characteristic angle $\left(\alpha_{n}\right)$ is a modal attribute that defines the phase lag between the characteristic currents $J_{n}$ and the tangential component of the characteristic electric field $E_{n}^{\text {tan }}$ on the surface $S$. It is an important metric parameter in the CM theory, which indicates the resonant behavior or the kind of stored energy of each mode [27]:

$$
\alpha_{n}=180^{\circ}-\tan ^{-1}\left(\lambda_{n}\right) \text {. }
$$

As $\alpha_{n}$ varies in the range $\left[90^{\circ}, 270^{\circ}\right.$ ], it provides an easy way to understand the mode behavior close to the resonance, especially if the mode presents narrow or large radiating bandwidth. CMs with a steep slope near $180^{\circ}$ for $\alpha_{n}$ curve present narrow bandwidth, whereas those with a smooth slope present larger bandwidth. The smoother the slope of $\alpha_{n}$ curve at $180^{\circ}$ is, the larger bandwidth the mode can exhibit.

According to the physical interpretation of eigenvalues, the characteristic modes can be also categorized using the characteristic angle $\alpha_{n}$ :

(i) If $\alpha_{n}=180^{\circ}$, the associated modes are resonant modes.

(ii) If $90^{\circ}<\alpha_{n}<180^{\circ}$, the associated modes are inductive modes.

(iii) If $180^{\circ}<\alpha_{n}<270^{\circ}$, the associated modes are capacitive modes.

2.4. CM Applied to Reconfigurable Antennas. As presented in Figure 1, the workflow of a reconfigurable antenna design based on a CMA is divided into three basic processes: simulation, fabrication, and measurement.

(i) Simulation: this step is fundamental, since all CMA carries out this step, which presents the following substeps:

(1) Understanding the structure: the first substep concerns the initial investigation to understand the behavior of the structure. The excitation of the structure is excluded at this stage. The CMA will find out which modes are consistently in or near resonance within the frequency range of interest. Besides, CMA can determine the attributes of a single mode or a combination of modes that are convenient for the target application.

(2) Correct mode excitation: as a second substep and once a mode or a combination of modes of interest is determined, an excitation for these modes must be conceived. Analysis of the surface current distribution associated to the modes of the structure is performed and can determine the appropriate antenna location feed. By means of the evaluation of the modal weighting coefficients, the analysis will find out how well the structure was able to achieve the selected modal behavior.

(3) Design parameter verification: the third substep deals with the verification action, where a chosen solver is used to calculate the performance parameters of the antenna such as $S$-parameters, radiation pattern, gain, directivity, or efficiency.

(ii) Fabrication: this step is practical and requires a good manufacturing ability that determines the accomplishment of the previous steps results. Additional parameters must be taken into consideration in the use of electronic components such as temperature, interference among bias wires, and feed.

(iii) Measurement: finally, this step validates the previous steps and shows the performance of the design structure.

\section{Geometry and Design of Rectangular Parallel Plates Based on Characteristic Mode Analysis}

Let us consider two rectangular metallic plates separated a distance $H=1.5 \mathrm{~mm}$, as shown in Figure 2. Two cases are analyzed, corresponding to two plates with the same dimensions $(W 1 \times L=62.5 \mathrm{~mm} \times 42 \mathrm{~mm})$ and two plates with a small variation in the length of the lower plate ( $W 2 \times L=68.5 \mathrm{~mm} \times 42 \mathrm{~mm})$.

Let us now analyze these two parallel plates applying the $\mathrm{CM}$ theory. Figure 3 presents the characteristic angle $\left(\alpha_{n}\right)$ analysis carried out over the $0-5 \mathrm{GHz}$ frequency range, for the plates with the same and with different dimensions. As seen in Figure 3, the observation of the curves at $\alpha_{n}=180^{\circ}$ shows that for both cases, there are two main resonant modes at the $2.5 \mathrm{GHz}$ band: antenna mode $J 1$ (where current flows in the same direction in both plates) and transmission line mode $J 1^{\prime}$ (where current flows in opposite direction in both plates), as depicted in Figure 4.

At resonance frequency, antenna mode $J 1$ and transmission line mode $J 1^{\prime}$ are presenting two different radiation patterns, as shown in Figure 5(a). However, by exciting the structure using a probe from the lower plate to the upper plate, a different combination of these two fundamental modes is excited by means of slightly expanding the dimensions of one plate. As shown in Figures 5(b) and 5(c), it can be observed that the resulting radiation pattern when exciting the structure with a probe feed gets varied from a bidirectional beam to a directional pattern, when slightly increasing the size of the lower plate.

The different modal contribution to the total radiated power in the two cases can be observed in Figures 6 and 7. As seen in Figure 6, mode $J 1^{\prime}$ (narrowband transmission line mode) dominates the total radiation power at $2.5 \mathrm{GHz}$ frequency band and leads to the bidirectional radiation pattern shown in Figure 5(b). Conversely, in Figure 7, when the dimensions of the parallel plates are slightly different, the total radiated power results in a combination of mode $J 1$ 


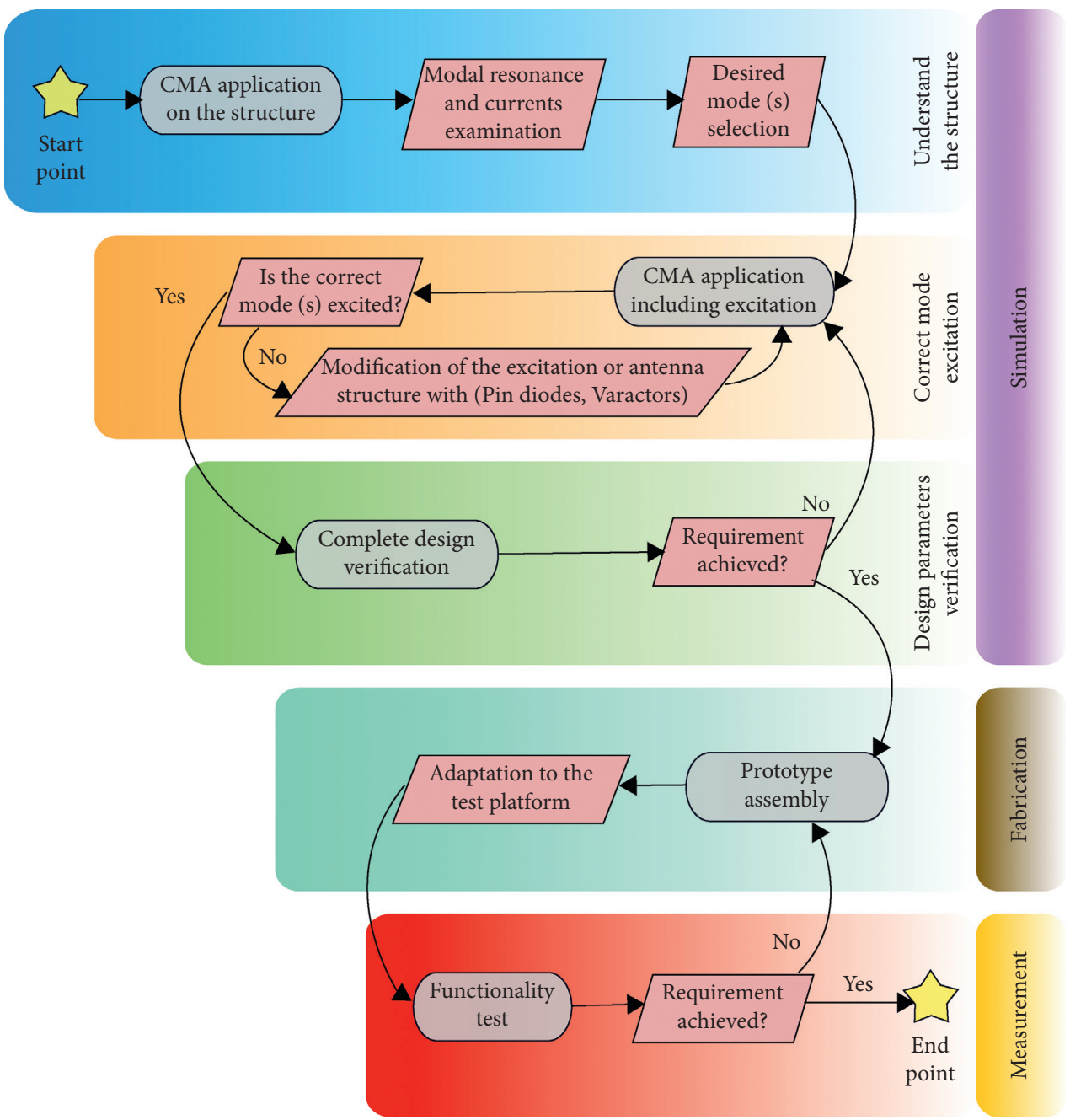

Figure 1: Workflow of a reconfigurable antenna design based on CMA.

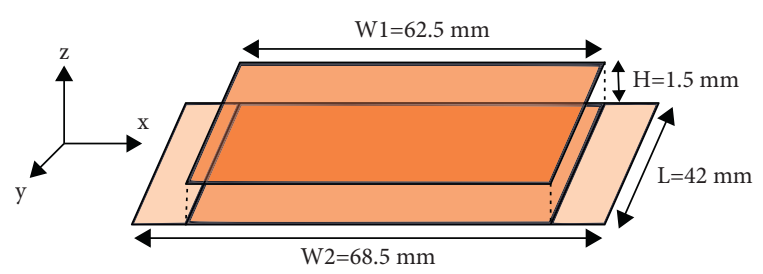

Figure 2: Geometry of the structure considered for the CMA.

(antenna mode) and $J 1^{\prime}$ (transmission line mode), and therefore this double mode excitation produces a directional pattern, as shown in Figure 5(c).

\section{Results and Discussion}

4.1. Reconfigurable Antenna Description. The proposed radiation pattern reconfigurable antenna is presented in Figure 8 and consists of a rectangular patch antenna printed on a Neltec substrate with a thickness $H$ of $1.524 \mathrm{~mm}$, $\varepsilon_{r}=2.2$, and tangent loss of 0.0009 . The dimensions of the

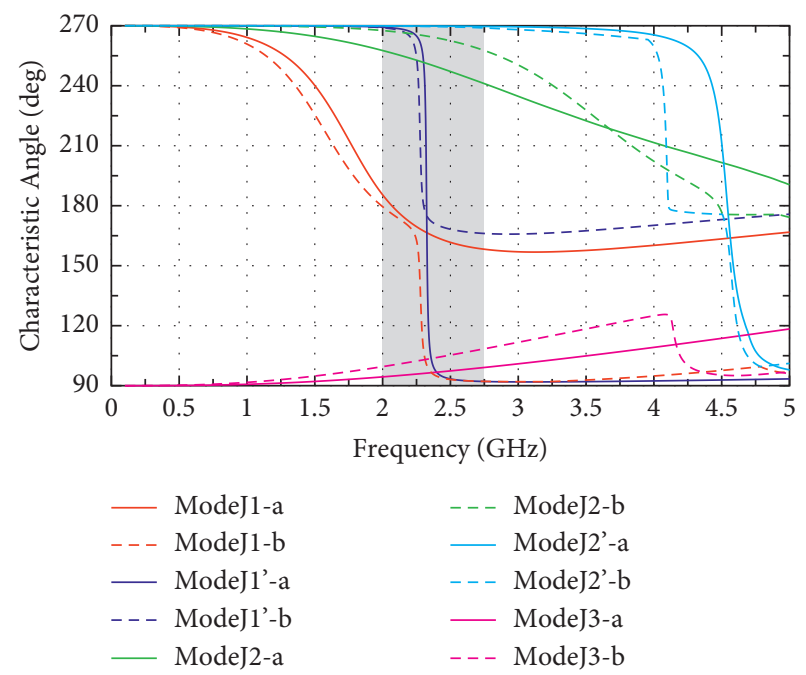

FIgURE 3: Characteristic angle associated to the first five modes of the structure shown in Figure 2. Mode-a case 1: parallel plates with the same dimensions. Mode-b case 2: parallel plates with different sizes. 


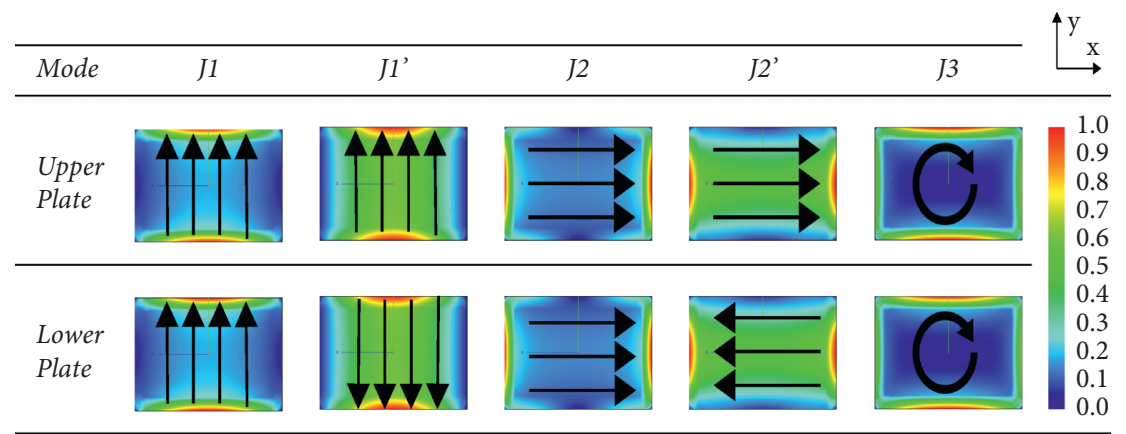

FIGURe 4: Current distribution associated to the first five modes of the structure shown in Figure 2, at the resonance frequency of each mode.
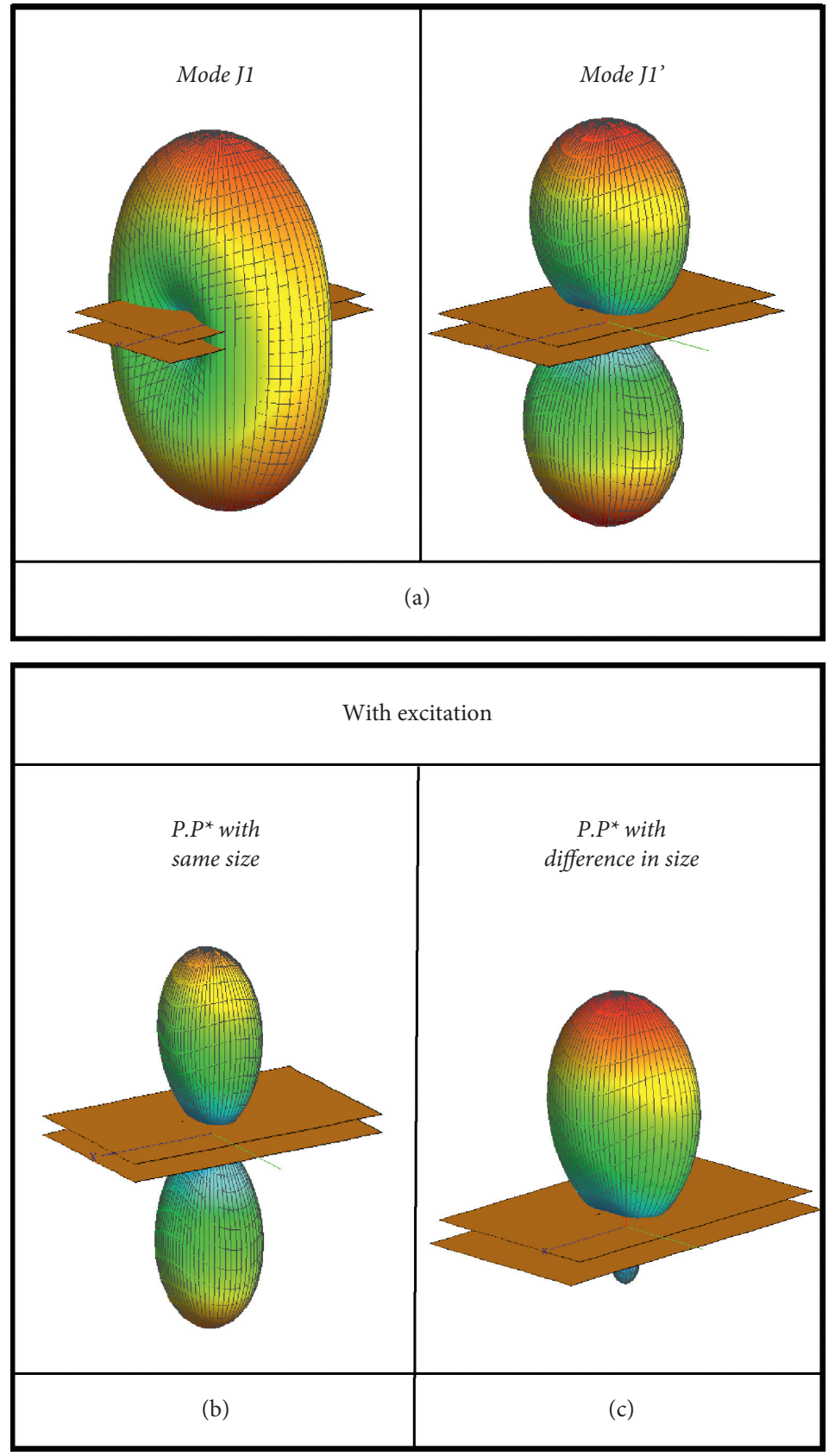

Total E-Field

8.0

7.2

6.4

5.6

4.8

4.0

3.2

2.4

1.6

0.8

0.0

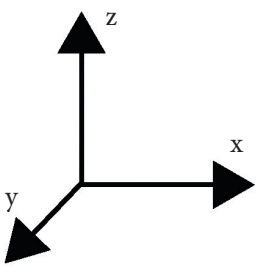

Total E-Field

8.0
7.2
6.4
5.6
4.8
4.0
3.2
2.4
1.6
0.8
0.0

Figure 5: Radiation pattern associated to (a) fundamental modes $J 1$ and $J 1^{\prime}$; (b) parallel plates (P.P ${ }^{*}$ ) with the same size; and (c) parallel

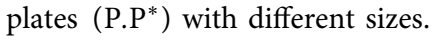




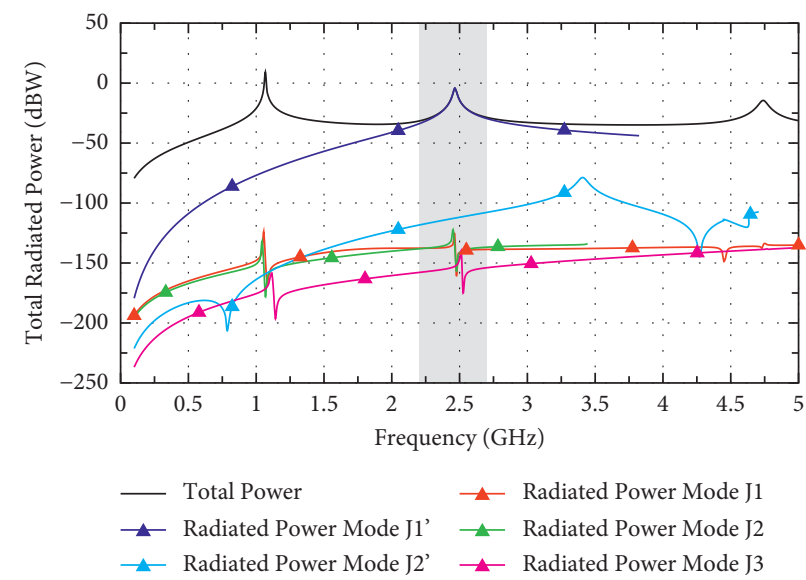

FIgURE 6: Total power and modal radiated power for two parallel plates with the same size.

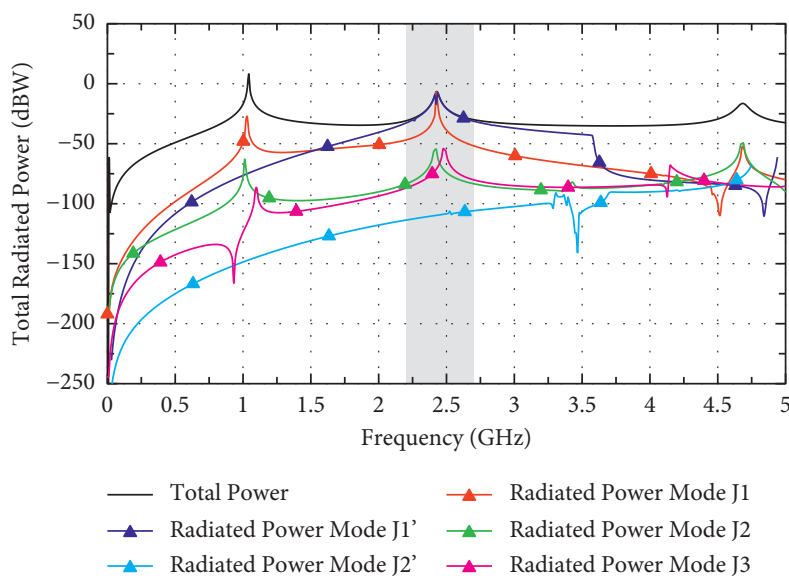

FIgURE 7: Total power and modal radiated power for two parallel plates with different sizes.

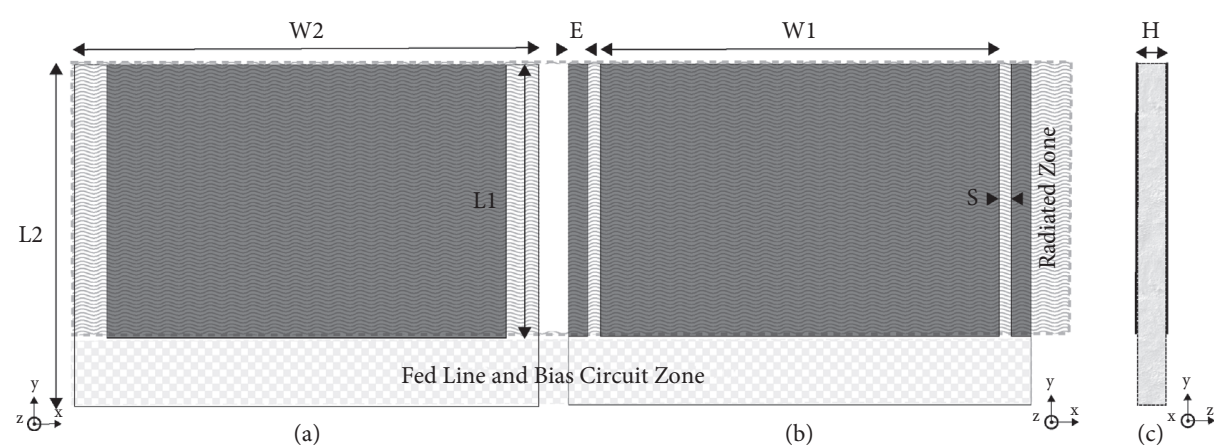

Figure 8: (a) Antenna's top view, (b) antenna's back view, and (c) antenna's lateral view.

structure are $W 1=41 \mathrm{~mm}, W 2=47.6 \mathrm{~mm}, L 1=28 \mathrm{~mm}$, $L 2=35 \mathrm{~mm}, S=1.3 \mathrm{~mm}$, and $E=2 \mathrm{~mm}$.

The idea is to implement an antenna consisting of two parallel plates, where the length of the lower plate is variable. Therefore, two PIN diodes are connecting the bottom plate to the lateral edges, as shown in Figure 9(c) (diodes D1 and
D2). The two PIN diodes states, labeled as ON and OFF, enable or disable the current to flow through the lateral edges; hence, and as shown in the modal analysis, the radiation pattern can get varied.

By switching from ON to OFF state, it is noted a shift in the operating frequency, due to a change in the input 


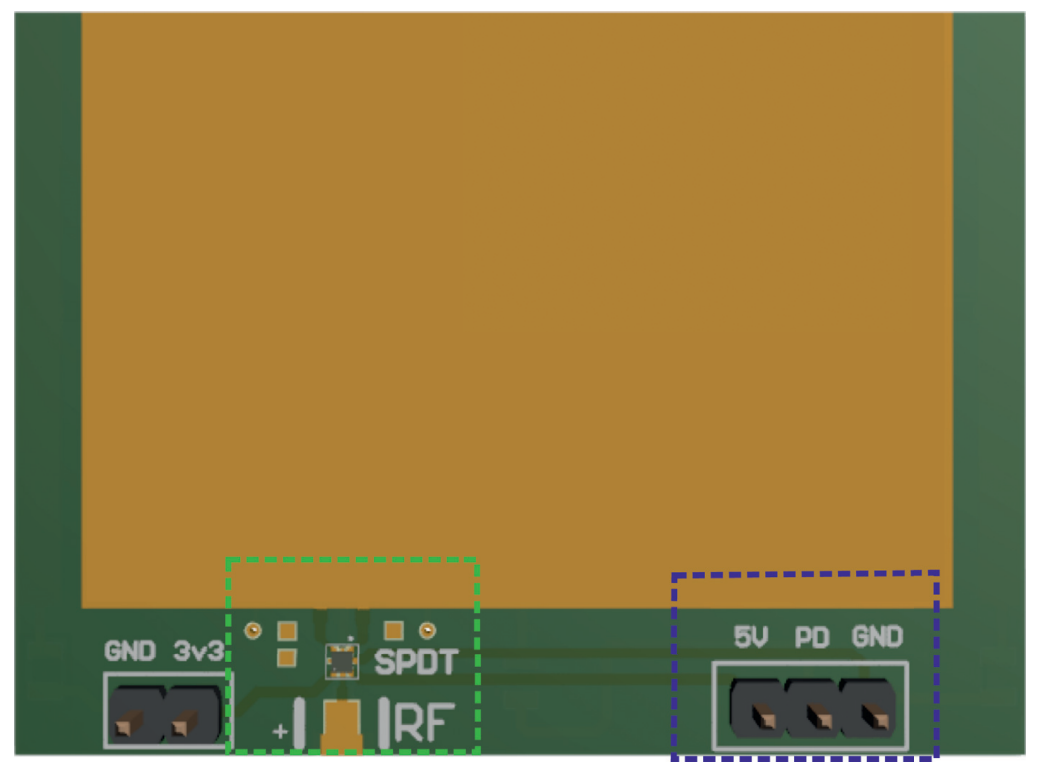

(a)

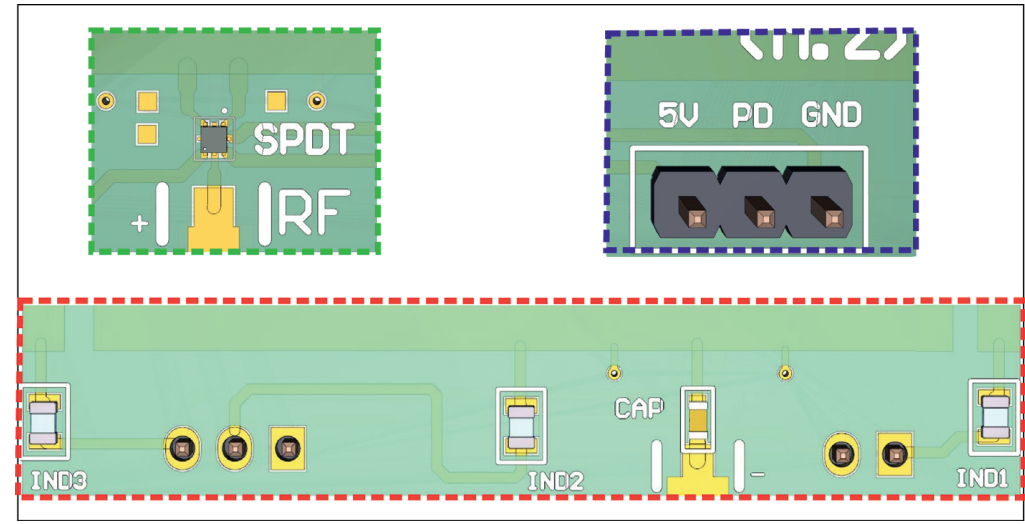

(b)

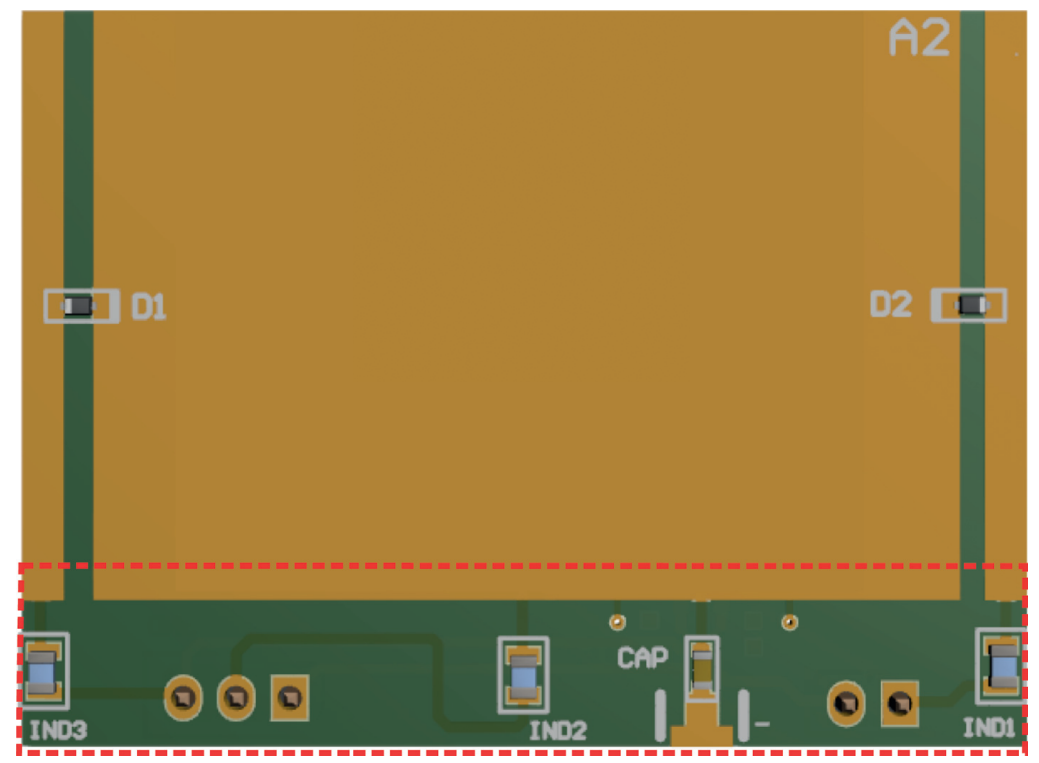

(c)

Figure 9: (a) Antenna's top view, (b) bias circuit areas, and (c) antenna's back view. 


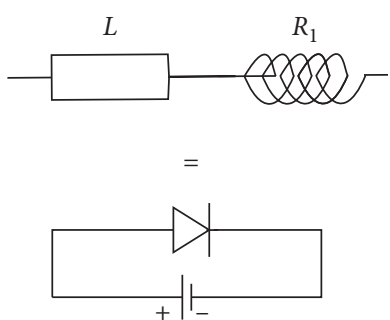

(a)

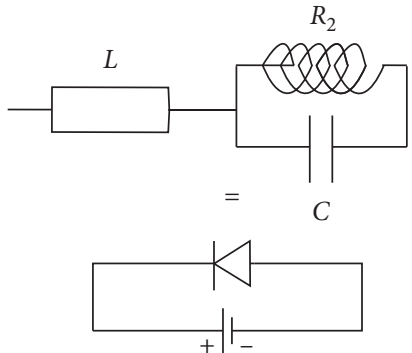

(b)

FIgURE 10: Equivalent circuit of the PIN diodes: (a) forward bias; (b) reverse bias.

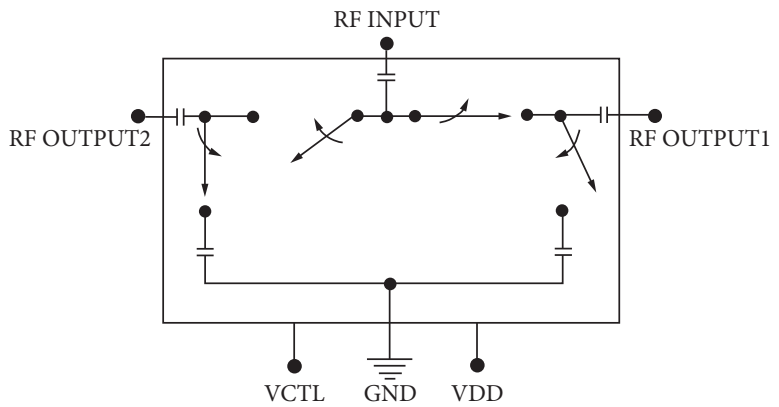

FIGURE 11: SPDT switch block diagram.

TABLE 1: Electronic components used in the proposed reconfigurable antenna.

\begin{tabular}{lcccc}
\hline Symbol & Value & Unit & Manufacturer & Comment \\
\hline D1 & BAR64-02V & - & Infineon & PIN diode \\
D2 & BAR64-02V & - & Infineon & PIN diode \\
SPDT & SKY13585-679LF & - & Skyworks & Switch \\
CAP & 12 & $\mathrm{pF}$ & Murata MLCC & DC block \\
IND1 & 68 & $\mathrm{nH}$ & Murata LQW & DC return \\
IND2 & 68 & $\mathrm{nH}$ & Murata LQW & RF choke \\
IND & 68 & $\mathrm{nH}$ & Murata LQW & DC return \\
\hline
\end{tabular}

impedance of the antenna. To settle this issue, a SPDT switch is added on the top plate. The aim of using a SPDT switch is to guarantee a common frequency operating band by switching the RF input signal to the adequate position related to the PIN diodes state, as shown in Figures 9(a) and 9(b).

4.2. Bias Circuit Configuration. Figure 10 shows the equivalent circuit of the PIN diode for forward and reverse biasing. The PIN diode model considered for the design is the Infineon BAR64-02V silicon PIN diode, with low insertion loss and fast switching time [28]. As shown in Figure 10, the equivalent circuit consists of an inductor and a parallel capacitor resistor. To obtain the first configuration (PIN diodes are reverse biased), the values of the elements are $(L=0.6 \mathrm{nH}, C=0.35 \mathrm{pF}$, and $R=3.3 \mathrm{k} \Omega)$. On the other hand, the element values of the second configuration (PIN diodes are forward biased) are $(L=0.6 \mathrm{nH}$ and $R=1.35 \Omega)$.
The SPDT switch (Figure 11) model adopted in the design is SKY13585-679LF [22], with a low insertion loss ( $0.5 \mathrm{~dB}$ typically at $2.45 \mathrm{GHz}$ ) for mode switching at ISM band. A remarkable advantage about using this model is that it has integrated DC blocking capacitors, so no external DC blocking capacitors are required.

As illustrated in Figure 9(b), to perform the DC bias supply to the PIN diodes D1 and D2, additional components are fitted in the feed line and bias circuit zone (see also Figure 8). A DC block capacitor CAP of $12 \mathrm{pF}$ is used in order to preserve the continuity of the RF current throughout the metal structure. The inductors IND1 and IND3 of $68 \mathrm{nH}$ provide the DC return path for the bias current. The DC voltage is isolated from the RF signal using a RF choke inductor IND2 of $68 \mathrm{nH}$. Table 1 summarizes the models and values used for the different components in the proposed design.

To meet the switches' biasing requirements, a platform based on a nanocontroller [29] and a battery is employed to 


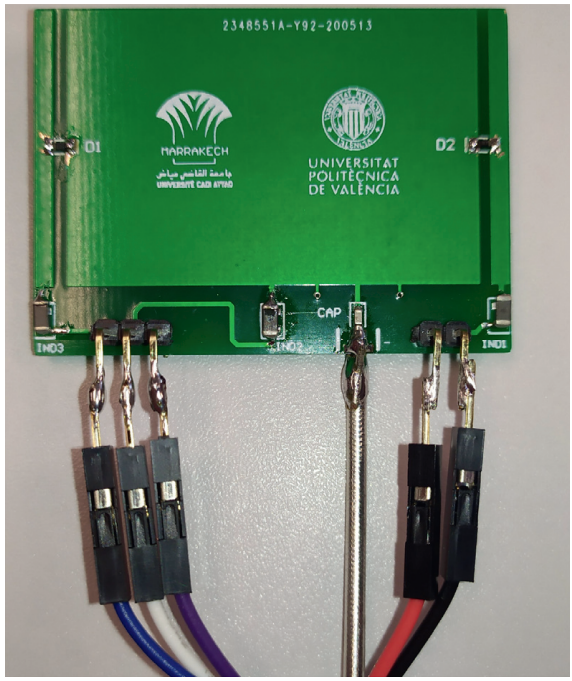

(a)

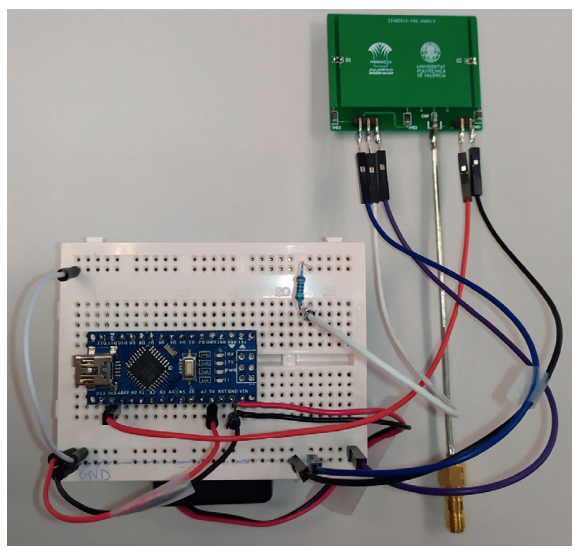

(c)

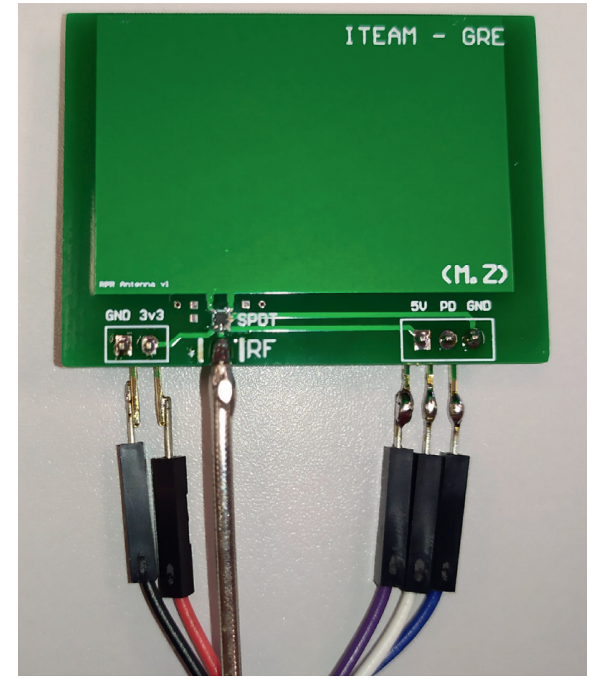

(b)

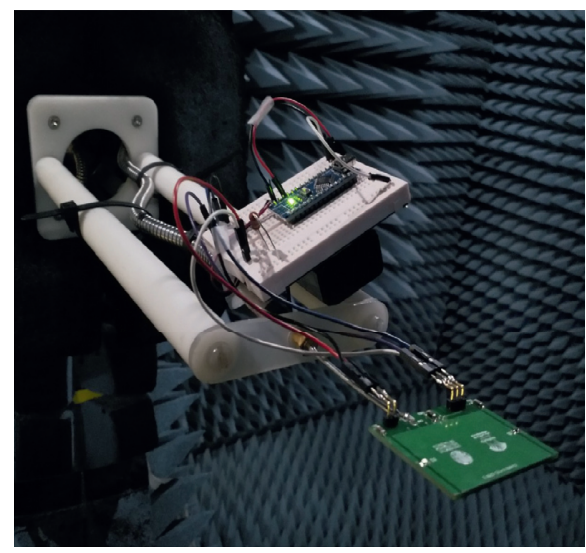

(d)

FIGURE 12: Final antenna prototype: (a) top view; (b) bottom view; (c) antenna with bias platform; (d) antenna in anechoic chamber.

TABLE 2: Reconfigurable antenna configuration: case 1 (bidirectional pattern) and case 2 (unidirectional pattern).

\begin{tabular}{lccc}
\hline Case & Mode(s) excited & PIN diodes & SPDT \\
\hline 1 & $J_{1}^{\prime}$ & OFF & VDD $=5 \mathrm{~V}, \mathrm{VCTL}=0 \mathrm{~V}$ \\
2 & $J_{1}^{\prime}+J_{1}$ & ON & VDD $=5 \mathrm{~V}, \mathrm{VCTL}=3.3 \mathrm{~V}$ \\
\hline
\end{tabular}

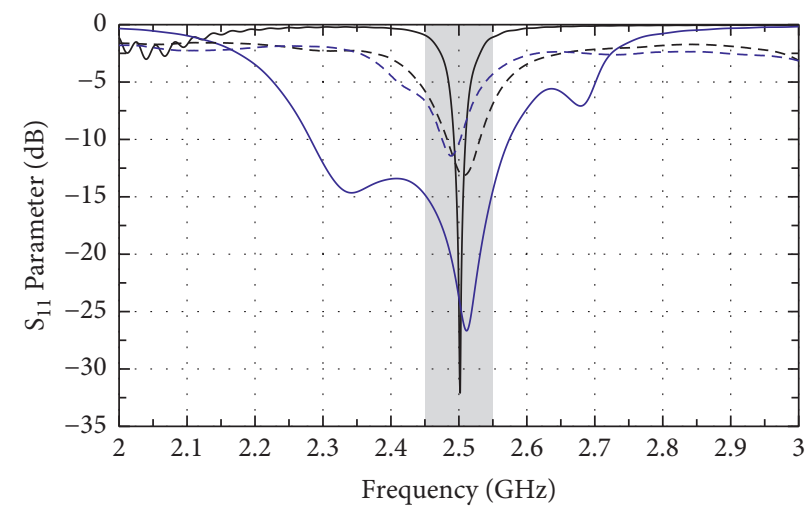

$\begin{array}{ll}-S_{11} \text { Simulated (case1) } & -S_{11} \text { Simulated (case2) } \\ ---S_{11} \text { Measured (case1) } & ---S_{11} \text { Measured (case2) }\end{array}$

FIGURE 13: Simulated and measured $S_{11}$ parameter for the ON/OFF cases presented in Table 2. 


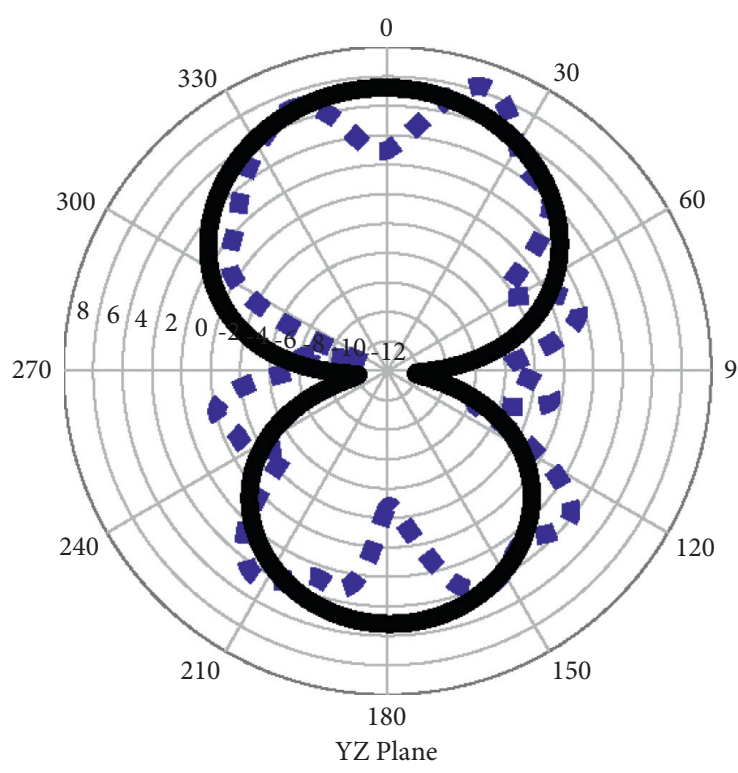

- - - Measured

- Simulated

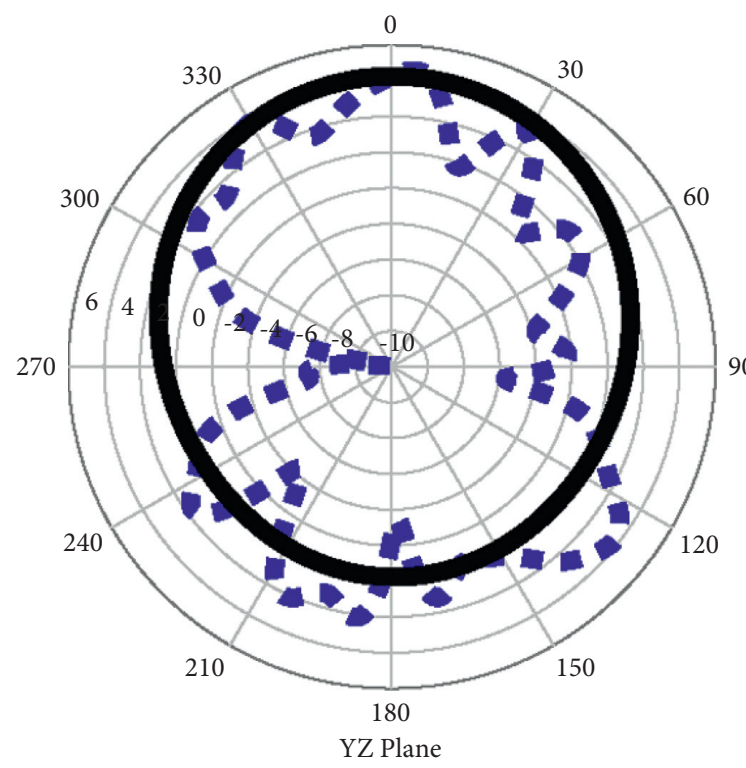

- - - Measured

Simulated

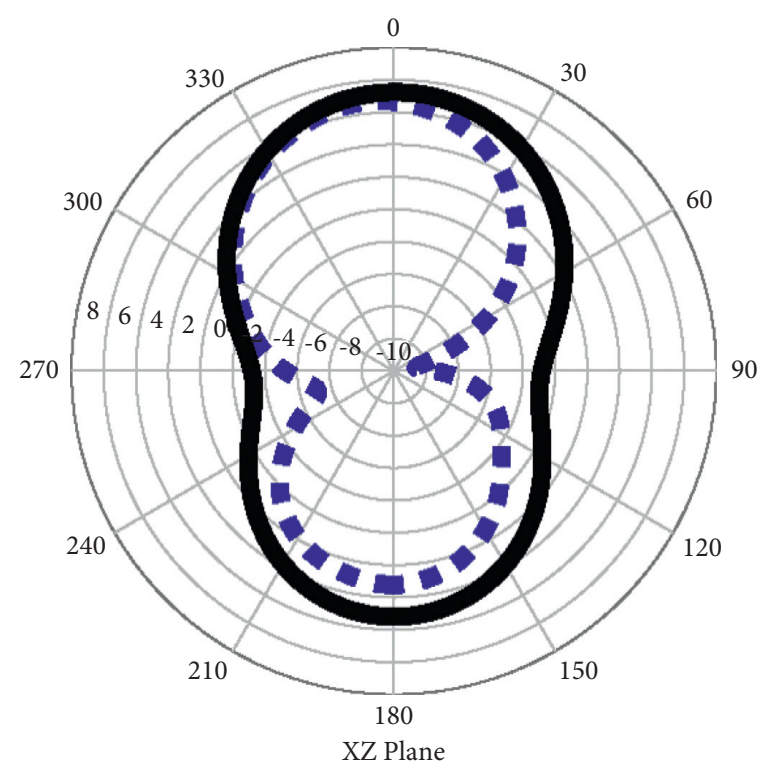

(a)

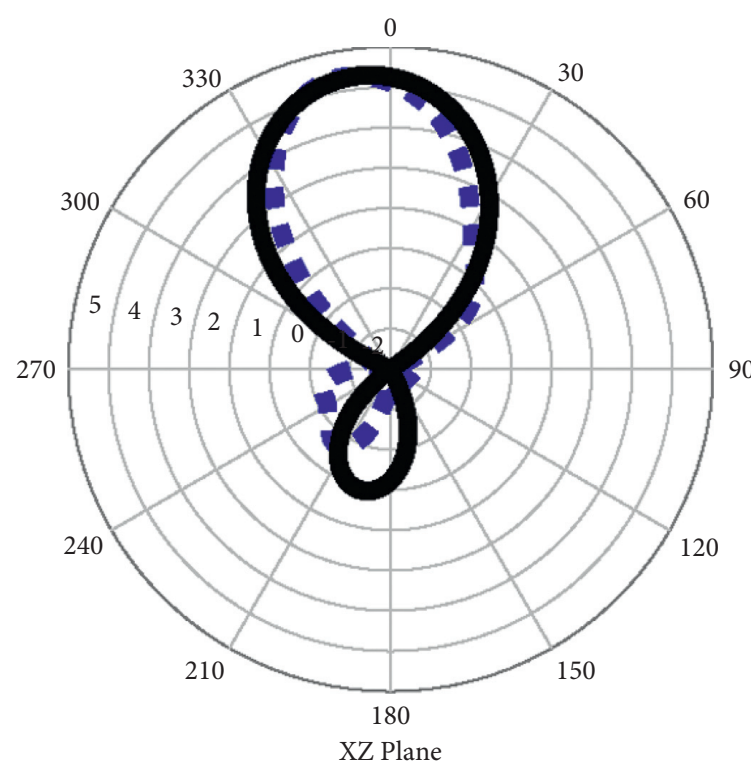

(b) achieve the bias signal corresponding to the two cases (case 1: bidirectional pattern and case 2: unidirectional pattern), as shown in Figure 12(c).

Table 2 shows the two configurations. To attain the first case, which consists of a bidirectional radiation pattern, the PIN diodes D1 and D2 were reverse biased and the SPDT INPUT pin (see SPDT block diagram in Figure 11) is connected to the RF OUTPUT1 by giving VDD and VCLT a supply voltage of $5 \mathrm{~V}$ and $0 \mathrm{~V}$, respectively. The second case corresponds to a directional radiation pattern achieved by forward biasing the PIN diodes D1 and D2, and the SPDT INPUT pin is connected to the RF OUTPUT2 by giving VDD and VCLT a supply voltage of $5 \mathrm{~V}$ and $3.3 \mathrm{~V}$, respectively.

4.3. Prototype and Measurement Results. The proposed radiation pattern reconfigurable antenna is fabricated and assembled with the electronic components as presented in Figure 12. In this prototype, connection wires are arranged 


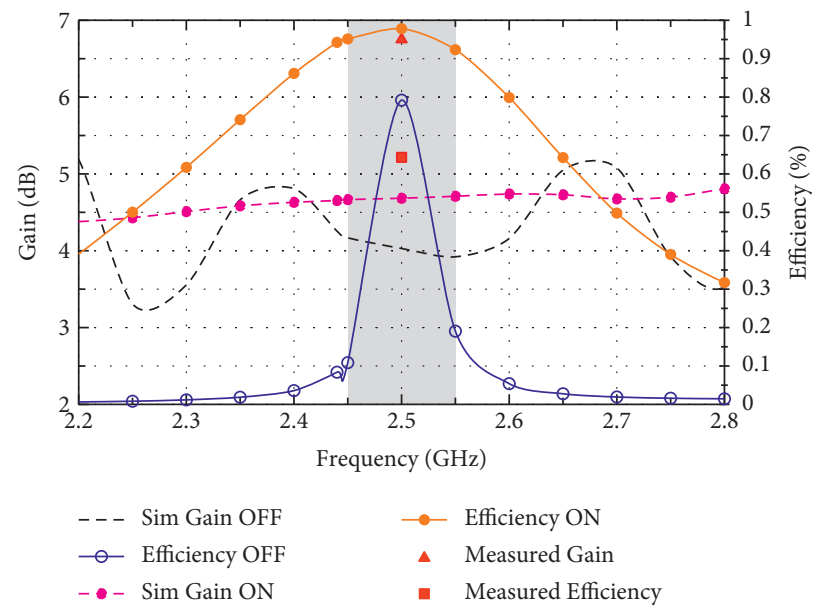

FIGURE 15: Antenna gain and efficiency.

TABle 3: Comparison of the proposed structure.

\begin{tabular}{|c|c|c|c|c|c|}
\hline Ref. & Beam type & Frequency $(\mathrm{GHz})$ & Number of beams & PIN diodes & Size $\left(\mathrm{mm}^{3}\right)$ \\
\hline [30] & Broadside and conical & $2.25-2.85$ & 3 & 2 & $170 \times 170 \times 17$ \\
\hline [31] & Omnidirectional & $3.4-3.8$ & 3 & 4 & $40 \times 30 \times 1.6$ \\
\hline$[32]$ & Sum and difference & $3.3-5.5$ & 2 & 4 & $144 \times 190 \times 0.8$ \\
\hline$[33]$ & Conical and broadside & $1.65-3.65$ & 2 & 6 & $140 \times 140 \times 6$ \\
\hline This work & Broadside and bidirectional & $2.28-2.58$ & 3 & 2 & $47.6 \times 35 \times 1.524$ \\
\hline
\end{tabular}

in an appropriate manner to minimize the perturbation on the radiation. Figure 13 shows the simulated versus measured $S_{11}$ parameter of the proposed radiation pattern reconfigurable antenna for both cases. From Figure 13, it is observed that simulation and measurement perfectly agree on a common band at $2.5 \mathrm{GHz}$. It can be noted that simulated $S_{11}$ parameter for case 2 shows a wide bandwidth. This is due to the fact that simulation files include ideal values for the lumped elements used for forward biasing the PIN diodes. The relative measured bandwidth at $-10 \mathrm{~dB}$ for case 1 and case 2 is, respectively, $60 \mathrm{MHz}$ and $25 \mathrm{MHz}$. However, measurement results meet the requirements, and a common operation frequency is achieved without changing physically the antenna structure.

$2 \mathrm{D}$ radiation pattern measurements of the reconfigurable antenna at $2.45 \mathrm{GHz}$ frequency band are plotted for both $X Z$ and $Y Z$ planes in Figure 14. As presented by the CMA in the first section, a reconfigurable radiation pattern is achieved. Turning the PIN diodes to ON state and SPDT to OUTPUT2 leads to a directional beam, and switching the PIN diodes to OFF state and SPDT to OUTPUT1 produces a bidirectional pattern. The maximum peak gain and the maximum radiation efficiency for both states have been measured as shown in Figure 15, reaching values up to $6.75 \mathrm{dBi}$ and $64.31 \%$ at $2.5 \mathrm{GHz}$, respectively. It should be noted that the efficiency is slightly low due to the diodes, SPDT switch, and particularly the nanocontroller platform that was incorporated in the measurement environment.

The performance of the proposed design is compared in Table 3 with some recently published designs featuring radiation pattern reconfigurable antenna performances. It is clear that the proposed antenna has fewer PIN diodes, which means less energy consumption and a low cost configuration. Moreover, it has the smallest size, except for the design presented in reference [31]. In comparison with the antenna having the same beam number $[30,31]$, the proposed antenna has different beams at the same resonant frequency which gives it toughness in terms of adaptation to the environment. Furthermore, the fabrication of our design is robust and the complexity is modest in comparison to the selected references.

\section{Conclusion}

The CM theory is able to provide a physical interpretation of the radiating behavior of an antenna. A radiation pattern reconfigurable antenna has been proposed in this paper based on the information provided by the CMA. The design of the antenna started from the modal analysis, in which the first case of two parallel plates with the same size shows a domination of mode $J 1^{\prime}$ and therefore a bidirectional pattern when the structure is excited. The second case, with a small difference in size in one plate, presents a unidirectional pattern, which arises from the contribution of two modes $J 1$ and $J 1^{\prime}$. After the analysis, a reconfigurable antenna with the same structure has been proposed with the aim of changing the radiation pattern, and two different patterns are achieved by switching ON/OFF state of the PIN diodes. By means of introducing a SPDT switch, the operating frequency at both states is adjusted to $2.45 \mathrm{GHz}$. The bias voltage used is low, which is a significant interest for IoT sensors and mobile stations. 


\section{Abbreviations}

CM: $\quad$ Characteristic mode

CMA: Characteristic mode analysis

IoT: Internet of Things

RA: Reconfigurable antenna

PIN: P-type layer, intrinsic layer, and N-type layer

SPDT: Single pole, double throw

DC: Direct current

VDD: DC supply voltage

VCTL: DC control voltage.

\section{Data Availability}

The data used to support the findings of this study are available from the corresponding author upon request.

\section{Conflicts of Interest}

The authors declare that they have no conflicts of interest.

\section{Acknowledgments}

This study was supported by the Spanish Ministry of Science and Innovation (Ministerio Ciencia e Innovación) under project no. PID2019-107885GB-C32 and Generalitat Valenciana under project no. AICO/2019/018.

\section{References}

[1] J. R. A. Garbacz, "Generalized expansion for radiated and scattered fields," Ph. D thesis, Ohio State University, Columbus, OH, USA, 1968.

[2] R. Garbacz and R. Turpin, "A generalized expansion for radiated and scattered fields," IEEE Transactions on Antennas and Propagation, vol. 19, no. 3, pp. 348-358, 1971.

[3] R. Harrington and J. Mautz, "Theory of characteristic modes for conducting bodies," IEEE Transactions on Antennas and Propagation, vol. 19, no. 5, pp. 622-628, 1971.

[4] E. Antonino-Daviu, M. Cabedo-Fabrés, M. Sonkki, N. Mohamed Mohamed-Hicho, and M. Ferrando-Bataller, "Design guidelines for the excitation of characteristic modes in slotted planar structures," IEEE Transactions on Antennas and Propagation, vol. 64, no. 12, pp. 5020-5029, 2016.

[5] M. Cabedo-Fabres, E. Antonino-Daviu, A. Valero-Nogueira, and M. Bataller, "The theory of characteristic modes revisited: a contribution to the design of antennas for modern applications," IEEE Antennas and Propagation Magazine, vol. 49, no. 5, pp. 52-68, 2007.

[6] J. Laheurte, Compact Antennas for Wireless Communications and Terminals. Theory and Design, John Wiley \& Sons, Inc, Hoboken, NJ, USA, 2011.

[7] H. Al-Tamimi and S. Mahdi, "A study of reconfigurable multiband Antenna for wireless application," International Journal of New Technology and Research, vol. 2, pp. 125-134, 2016.

[8] Z. Mahlaoui, E. Antonino-Daviu, A. Latif, and M. FerrandoBataller, "Design of a dual-band frequency reconfigurable patch antenna based on characteristic modes," International Journal of Antennas and Propagation, vol. 2019, Article ID 512532, 12 pages, 2019.
[9] X. Zhao and S. Riaz, "A dual-band frequency reconfigurable MIMO patch-slot antenna based on reconfigurable microstrip feedline," IEEE Access, vol. 6, pp. 41450-41457, 2018.

[10] G. Jin, M. Li, D. Liu, and G. Zeng, "A simple planar patternreconfigurable antenna based on arc dipoles," IEEE Antennas and Wireless Propagation Letters, vol. 17, no. 9, pp. 1664-1668, 2018.

[11] J. Zhang, S. Yan, and G. A. E. Vandenbosch, "Metamaterialinspired dual-band frequency-reconfigurable antenna with pattern diversity," Electronics Letters, vol. 55, no. 10, pp. 573-574, 2019.

[12] D. Chen, W. Yang, W. Che, Q. Xue, and L. Gu, "Polarizationreconfigurable and frequency-tunable dipole antenna using active AMC structures," IEEE Access, vol. 7, pp. 77792-77803, 2019.

[13] A. Bhattacharjee, S. Dwari, and M. K. Mandal, "Polarizationreconfigurable compact monopole antenna with wide effective bandwidth," IEEE Antennas and Wireless Propagation Letters, vol. 18, no. 5, pp. 1041-1045, 2019.

[14] E. González, J. Casanova-Chafer, A. Romero, X. Vilanova, J. Mitrovics, and E. Llobet, "LoRa sensor network development for air quality monitoring or detecting gas leakage events," Sensors, vol. 20, no. 21, p. 6225, 2020.

[15] M. Shirazi, J. Huang, T. Li, and X. Gong, "A switchablefrequency slot-ring antenna element for designing a reconfigurable array," IEEE Antennas and Wireless Propagation Letters, vol. 17, no. 2, pp. 229-233, 2018.

[16] C. Rick, "The nuts and bolts of tuning varactors," High Frequency Electronics, pp. 44-51, 2009.

[17] A. Zohur, H. Mopidevi, D. Rodrigo, M. Unlu, L. Jofre, and B. A. Cetiner, "RF MEMS reconfigurable two-band antenna," IEEE Antennas and Wireless Propagation Letters, vol. 12, pp. 72-75, 2013.

[18] I. F. Da Costa, S. A. Cerqueira, D. H. Spadoti, L. G. Da Silva, J. A. J. Ribeiro, and S. E. Barbin, "Optically controlled reconfigurable antenna array for mm-wave applications," IEEE Antennas and Wireless Propagation Letters, vol. 16, pp. 2142-2145, 2017.

[19] M. Konca and P. A. Warr, "A frequency-reconfigurable antenna architecture using dielectric fluids," IEEE Transactions on Antennas and Propagation, vol. 63, no. 12, pp. 5280-5286, 2015.

[20] A. Jouade, M. Himdi, A. Chauloux, and F. Colombel, "Mechanically pattern-reconfigurable bended horn antenna for high-power applications," IEEE Antennas and Wireless Propagation Letters, vol. 16, pp. 457-460, 2017.

[21] Z. Mahlaoui, E. Antonino-Daviu, A. Latif, and M. FerrandoBataller, "Radiation pattern reconfigurable antenna design using characteristic modes," in Proceedings of the 12th European Conference on Antennas and Propagation (EuCAP 2018), pp. 1-4, London, UK, April 2018.

[22] Skyworks Solutions, Inc, SKY13585-679LF: 1.0 to $6.0 \mathrm{GHz}$ SPDT Switch, Skyworks Solutions, Inc, Irvine, CA, USA, 2017, https://www.skyworksinc.com.

[23] FEKO Softwaer, "Solving connectivity, compatibility, and radar challenges," 2021, https://altairhyperworks.com/ product/FEKO.

[24] CST Studio Suite Software, "Software de simulación de campo electromagnético," 2021, https://www.3ds.com/es/productosy-servicios/simulia/productos/cst-studio-suite/.

[25] C. Yikai and W. Chao-Fu, Characteristic Modes: Theory and Applications in Antenna Engineering, John Wiley \& Sons, Inc., Hoboken, NJ, USA., 1 edition, 2015. 
[26] B. A. Austin and K. P. Murray, "The application of characteristic-mode techniques to vehicle-mounted NVIS antennas," IEEE Antennas and Propagation Magazine, vol. 40, no. 1, pp. 7-21, 1998.

[27] E. Newman, "Small antenna location synthesis using characteristic modes," IEEE Transactions on Antennas and Propagation, vol. 27, no. 4, pp. 530-531, 1979.

[28] A. G. Infineon Technologies, "Low signal distortion, surface mount RF PIN diode," 2018, https://www.infineon.com.

[29] Arduino, "User manual arduino nano (V2.3)," 2021, https:// www.arduino.cc/en/uploads/Main/ArduinoNanoManual23. pdf.

[30] W. Lin, H. Wong, and R. W. Ziolkowski, "Wideband patternreconfigurable antenna with switchable broadside and conical beams," IEEE Antennas and Wireless Propagation Letters, vol. 16, pp. 2638-2641, 2017.

[31] L. Han, C. Wang, W. Zhang, R. Ma, and Q. Zeng, "Design of frequency- and pattern-reconfigurable wideband slot antenna," International Journal of Antennas and Propagation, vol. 20187 pages, Article ID 678018, 2018.

[32] S.-A. Malakooti and C. Fumeaux, "Pattern-reconfigurable antenna with switchable wideband to frequency-agile bandpass/bandstop filtering operation," IEEE Access, vol. 7, pp. 167065-167075, 2019.

[33] X. Yang, H. Lin, H. Gu, L. Ge, and X. Zeng, "Broadband pattern diversity patch antenna with switchable feeding network," IEEE Access, vol. 6, pp. 69612-69619, 2018. 